\title{
Cardiac Electrophysiology Model Adjustment Using the Fusion of MR and Optical Imaging
}

\author{
D. Lepiller ${ }^{1}$, M. Sermesant ${ }^{1}$, M. Pop ${ }^{2}$, H. Delingette ${ }^{1}$, G.A. Wright ${ }^{2}$, \\ and N. Ayache ${ }^{1}$ \\ ${ }^{1}$ ASCLEPIOS Research Project, INRIA, Sophia Antipolis, France \\ ${ }^{2}$ Department of Medical Biophysics, University of Toronto, Sunnybrook Health \\ Sciences Centre, Toronto, Canada
}

\begin{abstract}
Despite important recent efforts in cardiac electrophysiology modelling, there is still a strong need for validating macroscopic models, that are well suited for diagnosis and treatment planning. In this paper we present a method to adjust the parameters of a macroscopic electrophysiology model on depolarisation and repolarisation maps obtained ex-vivo from optical imaging. With this imaging technique, optical fluorescence data are recorded with high spatial and temporal resolution on a large healthy porcine heart. A model of the myocardium is built from the MR images of the same heart, which also integrates the myocardial fibre orientation measured with DTI. We then present the first quantitative adjustment of a personalised volumetric model of the myocardium.
\end{abstract}

\section{Introduction}

In order to provide a better understanding of the mechanisms involved in cardiac arrhytmias, a variety of mathematical models have been developped for several decades to numerically simulate the cardiac electrophysiology at different scales. There are three main categories of such models. Ionic models [1] of the cardiac electrical activity describe the variation of ion concentrations across the membrane of cardiac cells. They may include a large number of variables and parameters. Their relative complexity usually leads to large computation times, but they can be precisely validated at the cell level. Far more simple are the Eikonal-based models [2, which only describe the time at which a depolarisation wave reaches a given point. At an intermediate level of complexity, we chose to use phenomenological models based on the transmembrane potential but without the different ion concentrations. They are based on PDEs that can describe with very few variables the coupled depolarisation and repolarisation processes.

This paper tackles the important issue of creating personalised electrophysiology models of the heart. Estimating the parameters of a model in order to decrease the error between the simulated variables and the observed ones is typically an inverse problem. Authors [3] focused recently on parameter estimation of the 63 variables Beeler-Reuter model at the cell level, but ionic models are not well suited for macroscopic model inversion due to the high number of parameters and variables to estimate. Theoretical results were recently demonstrated

D. Metaxas et al. (Eds.): MICCAI 2008, Part I, LNCS 5241, pp. 678 685, 2008.

(C) Springer-Verlag Berlin Heidelberg 2008 
on the estimation of the bidomain model parameters and anisotropy, but only on a $2 \mathrm{D}$ surface, and with synthetic data [4. On the other hand, the estimation of apparent electrical conductivity has been reported from clinical or experimental data based on Eikonal [5] and phenomenological models [6]. Although those pioneering works have reported good agreement between simulation and observations, they are somewhat limited since they only consider the depolarisation propagation on a surface and in some case [6] without taking into account the fibre orientation.

The objective of our work is to create a volumetric personalised electrophysiology model of the myocardium that takes into account the fibre orientation. Our approach relies on a tetrahedral mesh of the myocardium and estimates regionally two parameters in order to fit both the depolarisation and repolarisation isochrone maps. The parameter estimation is based on a calibration stage that takes advantage of existing analytical relationships in $1 \mathrm{D}$ between parameters and data. Furthermore, we rely on state of the art optical fluorescence imaging techniques to acquire ex-vivo isochrone maps on a pig heart. After applying different signal processing algorithms, dense maps can be retrieved. We then describe the creation of a computational personalised electrophysiology model from anatomical MR images, fibre orientation being extracted from subject specific Diffusion Tensor Imaging. Finally, we show the results of the estimation and discuss about the relevance of a synthetic model of fibre orientations.

\section{Simulation of the Transmembrane Potential}

The contraction of the heart muscle, the myocardium, is triggered by the depolarisation of the transmembrane potential. After depolarisation, a plateau phase begins during which the contraction develops ; this is the action potential duration $(a p d)$. The transmembrane potential then returns to a non-excited state (step called repolarisation). We use a phenomenological reaction-diffusion type model designed by Aliev-Panfilov [7], based on the transmembrane potential, to describe this propagation. It also takes into account the restitution phenomenon, i.e. the relation between heart rate and action potential duration. However, since we simulate and observe only one single cardiac cycle, a simplified repolarization equation, which neglects the description of the restitution curve, is used:

$$
\left\{\begin{array}{l}
\partial_{t} u=\operatorname{div}(D \nabla u)+k u(1-u)(u-a)-u z \\
\partial_{t} z=-\epsilon(k u(u-a-1)+z)
\end{array}\right.
$$

In this formulation, $u$ is a normalised transmembrane potential, and $z$ is a variable modelling the repolarisation. The diffusion term is controlled by the diffusion tensor $D$ which is similar to a physical conductivity. In the main direction of the tensor, this pseudo-conductivity is set to $d$ which is one of the parameters we adjust, and to $d / 2.5^{2}$ in the orthogonal directions 8. Parameter $k$ controls the repolarization, $a$ the reaction phenomenon and $\epsilon$ the coupling between the transmembrane potential and the repolarization variable. This system is solved over a volumetric tetrahedral mesh of the left and right ventricles using the finite 
elements method. The excitation is generated by imposing a potential on a small set of vertices for a few milliseconds. The time integration of the system is done with an explicit Euler scheme.

\section{Data Acquisition, Processing, and Fusion}

Optical Imaging. It is very challenging to acquire quantitative data that precisely reveals in vivo physiology. However, useful data can still be extracted from electrical or, as it is the case here, optical recordings. They consist of the instants when the depolarisation (and the repolarisation) occurs at specific locations of the heart. These data, called activation times, are enough to estimate the speed of the depolarisation front, and thus to adjust the pseudo-conductivity $d$, and to calibrate the parameter $a$ regarding the action potential duration, i.e. the difference between repolarisation and depolarisation times.

In this paper we performed the adjustments using optical recordings obtained in a healthy porcine heart. Large hearts are preferred for this work as they are close in size to human hearts. The explanted hearts were attached to a Langendorff perfusion system which permits to maintain the electrophysiological integrity of the hearts over 1-2 hours. The fluorescence dye (reflecting directly the changes of transmembrane potential) and the electromechanical uncoupler were injected into the perfusion line (more details are given in [9]). The hearts were paced with an electrode near the apex for $5 \mathrm{~ms}$. The fluorescence signals are captured with high temporal $(270 \mathrm{fps})$ and spatial $(<1 \mathrm{~mm})$ resolution, using a pair of CCD cameras (BrainVision, Jp). The signals giving the action potential waves at each pixel (Fig. 2a) were then filtered and further analysed to get a consistent map of activation times. The signal is scaled for each pixel between its baseline value and its maximum, cropping under the baseline which we got from segmenting the values into two clusters: the baseline being defined as the mean value of the lowest cluster. The scaled recording was then blurred by convolution with a 3D Gaussian, isotropic through space but wider in time (Fig. 2b). The depolarisation time is computed with the first derivative of each pixel signal, which presents a large peak when the depolarisation occurs. The repolarisation time is detected when the signal decreases $90 \%$ from its maximal value after depolarisation. Finally, the time maps for each cycle are reconstructed, stored as images and rectified based on the cameras calibration and stereoscopic parameters.

Optical and MR Data Fusion. At the end of the optical image acquisition, markers were put on the surfaces of the hearts, imaged with the CCD cameras, and the pig hearts scanned with a $1.5 \mathrm{~T}$ Signa GE MR scanner. It can provide a detailed anatomical description of both the geometry and the fibre orientation (Fig. 1a,b). The surfaces of the hearts were created using classical segmentation algorithms (thresholding, mathematical morphology, isosurface extraction) and volumetric meshes were generated with a meshing software (GHS3D, developed at INRIA) resulting in tetrahedral geometries of approximatively 75000 elements, with fibre orientation (Fig. 1 r). Several hearts were imaged this way. 


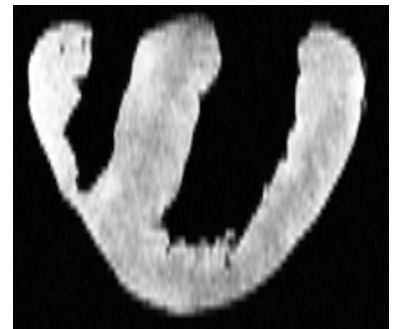

(a)

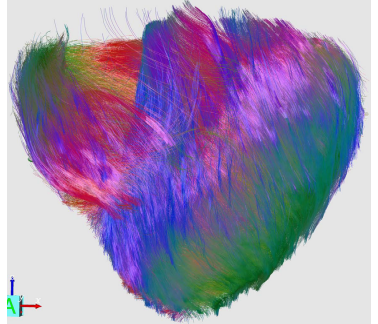

(b)

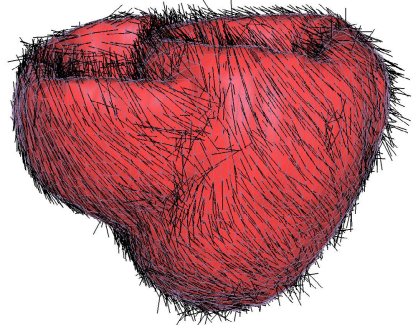

(c)

Fig. 1. MRI Data: (a) slice of the 3D volume used to create the myocardial mesh, (b) DTI fibre tracking, (c) generated volumetric mesh with assigned fibres (lateral views)

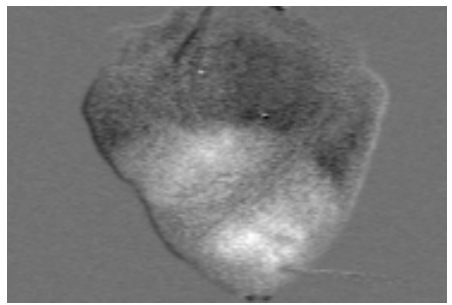

(a)

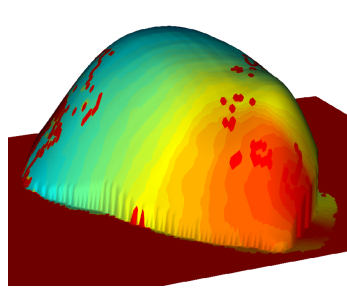

(b)

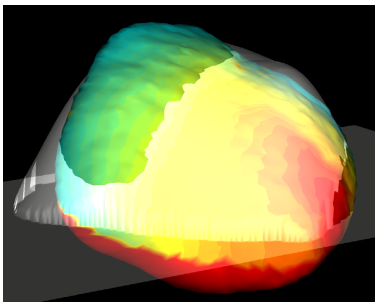

(c)

Fig. 2. Activation times measurement: (a) raw signal (anterolateral view) (b) isochrones computed from filtered signal on stereoscopic surface, (c) projection of the depolarisation times onto the MR derived mesh using registration (stereoscopic surface in transparency)

However, due to the complexity of the overall process, the full adjustment process was only done on one dataset.

The optical images recorded by the 2 CCD cameras were used to reconstruct the 3D surface of the heart using stereoscopy. Moreover, several opaque markers were glued onto the epicardium to provide a way to register the optical images with the MR images. We estimated a rigid transformation between the markers' optical and MR coordinates by minimizing the least-square difference. Each pixel of the optical recording corresponds to a vertex on the grid mesh which results from the stereoscopic reconstruction of the surface of the pig heart (further details can be found in [10]). Therefore the depolarisation front was spatially reconstructed, discretised on the mesh. We then projected these data onto the surface of the registered volumetric mesh from MR Imaging (results at Fig. 2r).

\section{Parameter Estimation}

Preparations. Setting constant parameters on the whole mesh would not allow us to take into account the local variations of the conduction velocity we observe. The left ventricle is therefore divided into 17 zones as defined by the American 
Heart Association and a similar division into 9 zones is applied to the right ventricle.

From the one dimensional analysis of the Aliev and Panfilov model, it can be shown that planar waves are solutions, and it provides a relationship between the conduction velocity $c$ and the first equation parameters [2] : $c=\sqrt{2 k d}(0.5-a)$. The same analysis was made for the relation between $a$ and the action potential duration $(a p d)$, which gives: $a p d=(a-1)^{2} / 4 a$. Again, this formula is only the restriction to one dimension since in the case of a three dimensional propagation, the curvature of the diffusion front affects its velocity. However this relation still tells us that the diffusion speed depends on several parameters and provides a probable relationship.

It seems more appropriate to first adjust the parameter $a$ from the action potential duration, since it does not depend on any other parameter, and then adjust the pseudo-conductivity parameter which would reflect the differences in conduction velocity in the tissue. In the Aliev-Panfilov model, $d$ and $k$ are the main parameters that affect this propagation speed. The $d$ parameter represents the diffusion properties of the myocardial tissue whereas $k$ accounts for the reaction of the ionic channels. Both can represent the variations of the electrical velocity of the tissue. As we only have one measure to adjust this speed, the depolarisation time, we chose to locally adjust the value of the parameter $d$, which represents this pseudo-conductivity, while keeping $k$ globally constant. Due to the imaging and registration errors, the activation times could present local variations. To avoid the amplification of this noise by the spatial derivatives of these depolarisation times, we smoothed the local speed computation by averaging it over a neighbouring area, weighted by the Euclidean distance between the vertices and the point where the speed is computed.

Calibration. The calibration method involves simulating several propagations on the mesh using a range of values for $d$ (resp. $a$ ), and to look at the corresponding speeds (resp. action potential duration). Then a function is fitted in the least square sense to these points in order to extract an analytical relationship between $d$ and $c$ (resp. $a$ and $a p d$ ), selected from the one dimensional analysis:

$$
c(d)=\alpha \sqrt{d}+\beta \quad \text { and } \quad \operatorname{apd}(a)=\frac{\gamma a^{2}+\delta a+\mu}{a}
$$

We added a constant $\beta$ to the relationship between $d$ and $c$ in order to better fit the numerical simulations. The idea of the calibration is also to take into account the numerical diffusion and discretisation errors in the calibration function.

This approximation is determined by computing the median conduction velocity (resp. apd) of each zone for each value of the parameter $d$ (resp. $a$ ) throughout a range of values. Then we minimise the least square difference to estimate the function parameters. Once this relationship is estimated, we can use it to initialise the value of each parameter $d_{i}$ (resp. $a_{i}$ ) for each zone, using the mean conduction velocity of each zone $\overline{c_{i}}$ (resp. the mean action potential duration $\left.a \bar{p} d_{i}\right)$ computed with the measured activation times: 


$$
d_{i}=\left(\frac{\overline{c_{i}}-\beta}{\alpha}\right)^{2} \quad \text { and } \quad a_{i}=\frac{\left(a \bar{p} d_{i}-\delta\right) \pm \sqrt{\left(a \bar{p} d_{i}-\delta\right)^{2}-4 \gamma \mu}}{2 \gamma}
$$

Regarding the apd, since there are usually two solutions, we choose the one within the range of acceptable values for parameter $a$.

Iterative adjustment. After the initialisation of the chosen parameter, we iteratively improve the model fitting, with a simple gradient descent algorithm. We minimise the following criteria on each zone: $J\left(d_{i}\right)=\left(\bar{c}_{i}-\hat{c}\left(d_{i}\right)\right)^{2}$ and $J\left(a_{i}\right)=$ $\left(a \bar{p} d_{i}-\operatorname{ap} d\left(a_{i}\right)\right)^{2}$ where $\hat{c}\left(d_{i}\right)$ is the median value of the simulated speed on the given zone using the parameter $d_{i}\left(\hat{a p} d\left(a_{i}\right)\right.$ defined alike). By minimising the differences between the simulated and measured depolarisation speeds for each zone instead of using the depolarisation times, we adjust all zones at the same time and thus considerably reduce the number of simulations to compute the gradient descent algorithm. Indeed, we can simplify the problem by assuming that the conduction velocity (and the action potential duration) of a zone is not strongly influenced by their neighbouring velocities.

\section{Results}

Action potential duration. Even if the initial value for $a$ was rather close to the measured values, the mean error on apd was still $44.6 \mathrm{~ms}(\approx 15 \%$ of APD) (Fig. 3b) before the adjustment process, $28.1 \mathrm{~ms}(\approx 9 \%)$ after calibration, and $21.5 \mathrm{~ms}(\approx 7 \%)$ after adjustment (Fig. 3r). The histograms of errors (Fig. 3a) clearly show this decrease. We find a shorter action potential duration on the right ventricle, as described in the literature.

Depolarisation times. Before calibrating the model, the mean absolute error on depolarisation times was $30.6 \mathrm{~ms}$ ( $\approx 17 \%$ of the depolarisation duration)

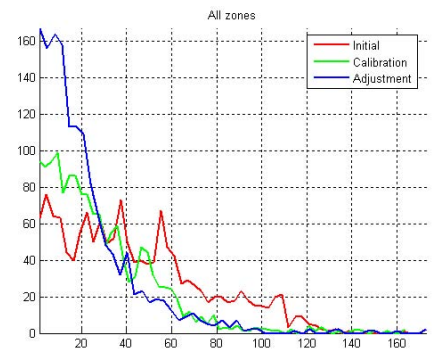

(a)

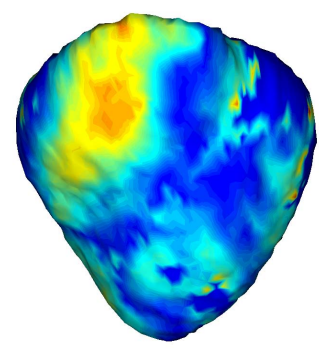

(b)

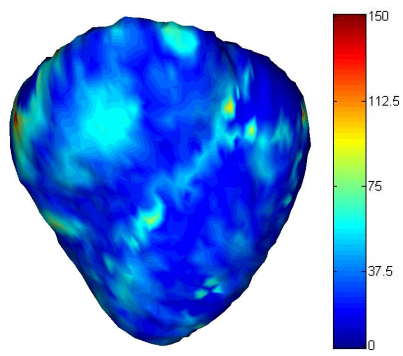

(c)

Fig. 3. (a) Histogram of errors on action potential duration across vertices (abscissa : error values in $m s$ ) and map of the error (b) before and (c) after adjustment (times in $m s$, anterolateral views) 


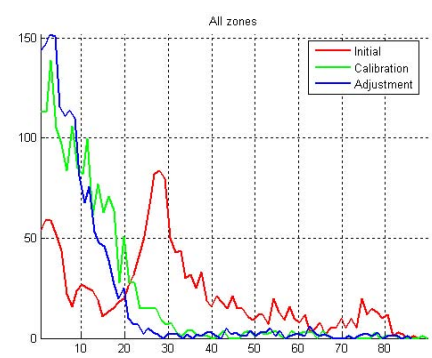

(a)

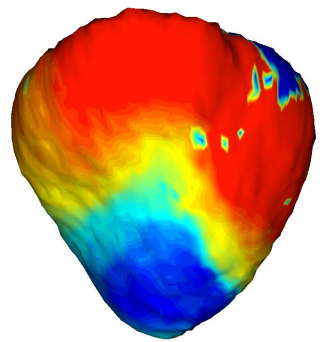

(b)

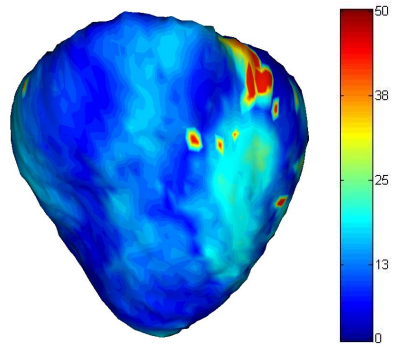

(c)

Fig. 4. (a) Histogram of errors on depolarisation time across vertices (abscissa : error values in $m s$ ) and map of the error (b) before and (c) after adjustment (times in $m s$, anterolateral views)

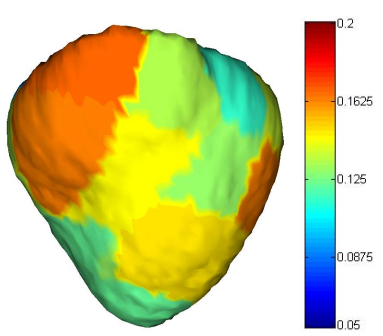

(a)

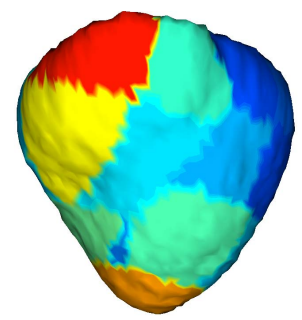

(c)

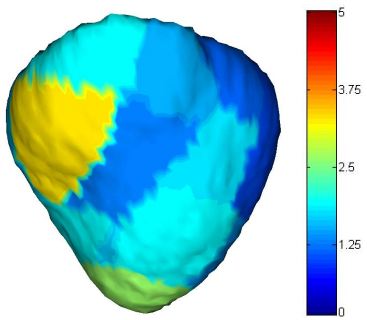

(d)

Fig. 5. Values of parameters after adjustment (a) of parameter a (c) of pseudoconductivity $d$ with measured fibre directions, (d) of pseudo-conductivity $d$ with synthetic model of fibres (anterolateral views)

(Fig. 4b). After the initial step of calibration, it decreased to $12.0 \mathrm{~ms}(\approx 7 \%)$ and the gradient descent algorithm further reduced it to $10.0 \mathrm{~ms}(\approx 5.5 \%)$ (Fig. 4 : $)$. With an error nearly divided by three, the calibration clearly improved the model accuracy and proved itself to be more useful than just an initialisation step of the iterative adjustment.

As in-vivo DTI is not yet available, we tested the relevance of a synthetic model of the fibre orientation, for clinical applications. The generation of synthetic fibres is based on the assumption that the elevation angle of the fibres on the endocardium and the epicardium is constant $\left(+90^{\circ}\right.$ and $-60^{\circ}$ respectively, to match the measured fibres), and that it varies linearly in between. Although the initial error on depolarisation times was higher than before $(37.9 \mathrm{~ms}$, $\approx 21 \%$ of the depolarisation duration), it decreased at nearly the same level than the previous adjustment final result with $10.7 \mathrm{~ms}(\approx 6 \%)$, showing that in this healthy case, the synthetic fibres provide a good alternative. Moreover, we can see with the comparison of estimated pseudo-conductivity maps with natural and synthetic fibres (Fig. 5. and 5d) that synthetic fibres provide a smoother variation. 


\section{Conclusion}

In this work we have verified that phenomenological models having a small number of parameters are well suited for personalisation, while keeping their ability to match the main characteristics of the action potential propagation: duration, speed, anisotropy. The processing of the activation times gives good results and the stereoscopic reconstruction and registration are accurate enough to convert the optical measurements into a smooth dataset of epicardial activation times. This leads to an estimation of parameters which considerably decreases the final error between model predictions and experimental measurements. Finally, the relevance of a synthetic anisotropy model was demonstrated. In the future, a multi-resolution scheme with an automatic recursive zone splitting should help the parameter optimisation process in convergence and provide a better segmentation of the locations where the conductivity varies significantly.

\section{References}

1. Noble, D.: A modification of the hodgkin-huxley equations applicable to purkinje fibre action and pace-maker potentials. J. Physiol. 160, 317-352 (1962)

2. Keener, J., Sneyd, J.: Mathematical Physiology. Springer, Heidelberg (1998)

3. Dokos, S., Lovell, N.H.: Parameter estimation in cardiac ionic models. Progress in Biophysics and Molecular Biology 85(2-3), 407-431 (2004)

4. Sadleir, R., Henriquez, C.: Estimation of cardiac bidomain parameters from extracellular measurement: two dimensional study. Annals of Biomedical Engineering 34(8), 1289-1303 (2006)

5. Chinchapatnam, P., Rhode, K., King, A., Gao, G., Ma, Y., Schaeffter, T., Hawkes, D., Razavi, R., Hill, D., Arridge, S., Sermesant, M.: Anisotropic wave propagation and apparent conductivity estimation in a fast electrophysiological model: Application to XMR interventional imaging. In: Ayache, N., Ourselin, S., Maeder, A. (eds.) MICCAI 2007, Part I. LNCS, vol. 4791, pp. 575-583. Springer, Heidelberg (2007)

6. Moreau-Villéger, V., Delingette, H., Sermesant, M., Ashikaga, H., McVeigh, E., Ayache, N.: Building maps of local apparent conductivity of the epicardium with a 2D electrophysiological model of the heart. IEEE Transactions on Biomedical Engineering 53(8), 1457-1466 (2006)

7. Aliev, R., Panfilov, A.: A simple two-variable model of cardiac excitation. Chaos, Solitons \& Fractals 7(3), 293-301 (1996)

8. Hsu, E., Muzikant, A., Matulevicius, S., Penland, R., Henriquez, C.: Magnetic resonance myocardial fiber-orientation mapping with direct histological correlation. American Journal of Physiology 274, 1627-1634 (1998)

9. Pop, M., Sermesant, M., Chung, D., Liu, G., McVeigh, E., Crystal, E., Wright, G.: An experimental framework to validate 3D models of cardiac electrophysiology via optical imaging and MRI. In: Sachse, F.B., Seemann, G. (eds.) FIHM 2007. LNCS, vol. 4466, pp. 100-109. Springer, Heidelberg (2007)

10. Chung, D., Pop, M., Sermesant, M., Wright, G.: Stereo reconstruction of the epicardium for optical fluorescence imaging. In: MICCAI Workshop on Biophotonics Imaging for Diagnostics and Treatment, pp. 33-40 (2006) 